\title{
Determinants of variations in coronary revascularization practices
}

\author{
Jack V. Tu MD PhD, Dennis T. Ko MD MSc, Helen Guo MSc, Janice A. Richards RN BA, Nancy Walton RN PhD, \\ Madhu K. Natarajan MD MSc, Harindra C. Wijeysundera MD, Derek So MD, David A. Latter MD, \\ Christopher M. Feindel MD, Kori Kingsbury MSN, Eric A. Cohen MD; for the Cardiac Care Network \\ of Ontario's Variations in Revascularization Practice in Ontario Working Group
}

See related commentary by Holmes and Rihal at www.cmaj.ca/lookup/doi/10.1503/cmaj.111946

\section{- ABSTRACT}

Background: The ratio of percutaneous coronary interventions to coronary artery bypass graft surgeries ( $\mathrm{PCl}: \mathrm{CABG}$ ratio) varies considerably across hospitals. We conducted a comprehensive study to identify clinical and nonclinical factors associated with variations in the ratio across 17 cardiac centres in the province of Ontario.

Methods: In this retrospective cohort study, we selected a population-based sample of 8972 patients who underwent an index cardiac catheterization between April 2006 and March 2007 at any of 17 hospitals that perform invasive cardiac procedures in the province. We classified the hospitals into four groups by $\mathrm{PCl}$ :CABG ratio (low [<2.0], low-medium [2.0-2.7], mediumhigh [2.8-3.2] and high [>3.2]). We explored the relative contribution of patient, physician and hospital factors to variations in the likelihood of patients receiving $\mathrm{PCl}$ or CABG surgery within 90 days after the index catheterization.

Results: The mean PCI:CABG ratio was 2.7 overall. We observed a threefold variation in the ratios across the four hospital ratio groups, from a mean of 1.6 in the lowest ratio group to a mean of 4.6 in the highest ratio group. Patients with single-vessel disease usually received $\mathrm{PCI}$ $(88.4 \%-99.0 \%)$ and those with left main artery disease usually underwent CABG $(80.8 \%-$ $94.2 \%)$, regardless of the hospital's procedure ratio. Variation in the management of patients with non-emergent multivessel disease accounted for most of the variation in the ratios across hospitals. The mode of revascularization largely reflected the recommendation of the physician performing the diagnostic catheterization and was also influenced by the revascularization "culture" at the treating hospital.

Interpretation: The physician performing the diagnostic catheterization and the treating hospital were strong independent predictors of the mode of revascularization. Opportunities exist to improve transparency and consistency around the decision-making process for coronary revascularization, most notably among patients with non-emergent multivessel disease.
Competing interests: Derek So has received financial support from Abbott Vascular and Eli Lilly. Christopher Feindel has received financial support from Edwards Lifesciences. Eric Cohen has received financial support from Abbott Vascular, Medtronic and Boston Scientific. The other authors have no financial conflicts of interest to declare.

Members of the Variations in Revascularization Practice in Ontario Working Group appear at the end of the article.

This article has been peer reviewed.

Correspondence to:

Dr. Jack V. Tu,

tu@ices.on.ca

CMAJ 2012. DOI:10.1503 /cmaj.111072
$\mathrm{L}$ arge inter-regional and inter-hospital variations exist in the ratio of percutaneous coronary intervention (PCI) procedures to coronary artery bypass graft (CABG) surgeries performed in many countries, but the reasons for these variations are uncertain. ${ }^{1-3}$ Bypass surgery was the first method of coronary revascularization to be developed. ${ }^{4}$ The less-invasive alternative of PCI was developed initially to treat single-vessel disease. However, advances in PCI technology (e.g., bare-metal stents and, later, drug-eluting stents) combined with increased operator experience have led to its use for a broader list of indications, including multivessel disease and acute coronary syndromes. ${ }^{5-7}$

In Ontario, Canada's most populous province, the overall PCI:CABG ratio has steadily increased, from 1.6 in 2001 to 2.7 in 2006 (unpublished data available from the authors upon request); similar increases have been observed in other jurisdictions. ${ }^{1,28}$ Although the change in ratio has been driven in part by expanded use of urgent PCI for acute myocardial infarction (MI), increased use of PCI in patients with multivessel disease has likely also been a contributing factor. This application of $\mathrm{PCI}$ is more controversial, because several studies, including the recent randomized SYNTAX (Synergy Between Percutaneous Coronary Intervention with TAXUS and Cardiac Surgery) trial, have shown that long-term outcomes of certain patients with multivessel disease were better with $\mathrm{CABG}$ surgery than with PCI. ${ }^{9-13}$

In addition to an overall increase in the PCI:CABG ratio, the amount of variation in the 
ratio across cardiac centres in Ontario has also steadily increased over time, with more than a threefold regional variation observed in 2006 (unpublished data available from the authors upon request). This degree of variation has raised concerns among some policy-makers and clinicians as to why such striking variations exist in Ontario's universal health care system. To address this issue, we conducted a comprehensive study to identify clinical and nonclinical factors associated with variations in the PCI:CABG ratio across the province's 17 cardiac centres.

\section{Methods}

\section{Study setting and design}

The Variation in Revascularization Practices in Ontario study was conducted by the Cardiac Care Network of Ontario in partnership with the Institute for Clinical Evaluative Sciences and the 17 cardiac centres in Ontario that provide invasive cardiac procedures. In 2006, 11 of the cardiac centres performed both PCI and CABG surgery, 1 performed PCI only (Rouge Valley Health System, Toronto, Ont.), and 5 performed cardiac catheterization only (Hôtel-Dieu Grace Hospital in Windsor, Peterborough Regional Health Centre, Sault Area Hospital, Thunder Bay Regional Health Sciences Centre and Toronto East General). (All 17 centres are listed in Box 1.)

\section{Chart audit}

We conducted a retrospective chart review of a population-based sample of 8972 patients who

\section{Box 1: Cardiac centres included in the study}

A Hôtel-Dieu Grace Hospital (Windsor)

B London Health Sciences Centre

C Thunder Bay Regional Health Sciences Centre

D St. Mary's General Hospital (Kitchener)

E Peterborough Regional Health Centre

F Rouge Valley Health System (Toronto)

G Trillium Health Centre (Mississauga)

H Hamilton Health Sciences

I Southlake Regional Health Centre (Newmarket)

J Toronto East General Hospital

K University Health Network (Toronto)

L Kingston General Hospital

M Sunnybrook Health Sciences Centre (Toronto)

N St. Michael's Hospital (Toronto)

O University of Ottawa Heart Institute

P Sudbury Regional Hospital (now Health Sciences North)

Q Sault Area Hospital underwent an index cardiac catheterization from April 2006 to March 2007 at any of the 17 cardiac hospitals in the province. The study was limited to patients whose primary indication for catheterization was coronary artery disease and who had not had a cardiac procedure in the year preceding the index catheterization. The patients reviewed were randomly sampled from the Cardiac Care Network of Ontario's master coronary angiography database; they represented about $20 \%$ of all patients who had angiograms in the province in 2006.

Experienced cardiology research nurses abstracted detailed clinical information from the hospital charts. The information collected was predetermined on the basis of a comprehensive set of patient characteristics and clinical management practices hypothesized to potentially influence revascularization decisions regarding PCI or $\mathrm{CABG}$.

\section{Determination of procedure ratios and coronary anatomy}

Clinical information from the hospital charts was linked to data from the Cardiac Care Network of Ontario's procedure database on PCI or CABG procedures performed within 90 days after the index catheterization. This database has been shown to be a valid source of procedural information. ${ }^{14}$ Only Ontario residents with a valid health card number were included in the study, which resulted in complete follow-up for the study cohort. All procedures were attributed to the centre where the index cardiac catheterization was performed, and a corresponding PCI: $\mathrm{CABG}$ ratio was determined for each centre. The participating hospitals were classified into four categories by ratio (low $[<2.0]$, low-medium [2.0-2.7], medium-high [2.8-3.2] and high [>3.2]) such that there were similar numbers of hospitals (four or five) in each category.

The coronary anatomy for each patient was determined from information abstracted from the coronary angiographer's procedure note or the catheterization anatomy procedure diagram, or both. Significant stenoses were defined as blockage of at least $70 \%$ in one or more major epicardial vessels or side branches, or blockage of at least $50 \%$ in the left main coronary artery. Mild disease was defined as evidence of coronary artery disease below these thresholds. Coronary anatomy and level of stenosis were validated as part of a substudy and indicated very good reliability when compared with a blinded interpretation by an interventional cardiologist (unpublished data available from the authors upon request). Patients who had undergone previous CABG surgery were classified in a separate anatomic category. 
The clinical indications for the index catheterization were classified into one of five categories: elective; unstable angina; non-STsegment elevation MI; non-emergent STsegment elevation MI; and emergent ST-segment elevation MI. Emergent ST-segment elevation MI referred to instances in which the catheterization was performed emergently for primary PCI, rescue PCI or cardiogenic shock. Non-emergent ST-segment elevation MI referred to other instances (e.g., routine catheterization after fibrinolytic therapy). Emergent cases were included in the initial calculation of overall hospital PCI:CABG ratios because variations in the proportion of these cases could influence the overall ratio. However, we excluded them from the more detailed analyses of factors associated with interhospital variations in procedure ratios, because these patients typically undergo PCI rather than CABG surgery.

\section{Statistical analysis}

All univariable descriptive analyses of the study data were performed using $t$ tests for continuous variables and $\chi^{2}$ tests for dichotomous variables. Multilevel (three-level) logistic regression models were developed to assess which patient, physician and hospital factors most influenced the choice of PCI over CABG surgery. ${ }^{15} \mathrm{We}$ compared the PCI:CABG ratios in different categories of coronary anatomy against the overall hospital ratio using a linear regression model in which the overall ratio was the dependent vari- able and the PCI:CABG ratios in the anatomy categories were the independent variables. We used backward stepwise regression to eliminate variables from the model whose $p$ values were greater than 0.05 . We used the $\mathrm{C}$-statistic to measure the discriminatory power of the logistic regression models and the $R^{2}$ statistic to measure the predictive power of the linear regression models. All $p$ values below 0.05 were considered statistically significant.

\section{Ethics approval}

The research ethics boards at each participating centre reviewed the study design, and a waiver of informed consent for collecting the study data was approved by each board. ${ }^{16}$

\section{Results}

The mean overall PCI:CABG ratio was 2.7. We observed a threefold variation in the ratios across the four hospital ratio groups, from a mean of 1.6 in the lowest ratio group to a mean of 4.6 in the highest ratio group. The ratios across the individual hospitals varied fivefold, from 1.3 to 6.1 (Figure 1).

The patients who underwent cardiac catheterization were fairly similar across the four ratio categories for most baseline characteristics (Table 1). Patients at hospitals in the highest ratio category were more likely to have had a previous PCI than patients in other hospitals $(p<0.001)$. Although the proportions of patients with emer-

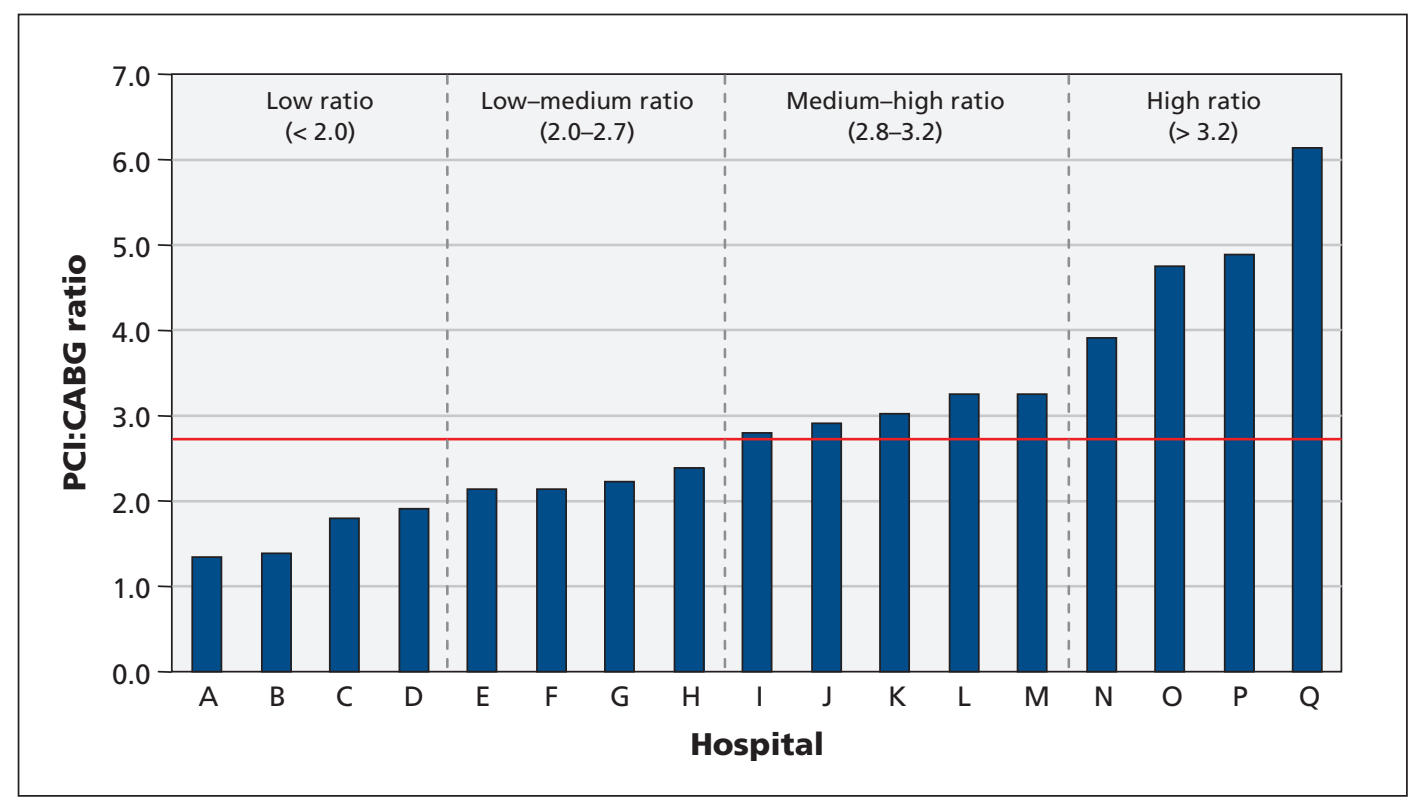

Figure 1: Distribution of hospitals by ratio of PCI:CABG procedures performed within 90 days after index cardiac catheterization at that hospital. For analysis, hospitals were classified into four categories of ratios such that there were similar numbers of hospitals (four or five) in each category. The red line represents the mean $\mathrm{PCl}$ :CABG ratio (2.7). CABG = coronary artery bypass graft, $\mathrm{PCl}=$ percutaneous coronary intervention. The names of the hospitals appear in Box 1. 
Table 1: Characteristics of patients, physicians and hospitals in which index cardiac catheterizations were performed between April 2006 and March 2007, by category of hospital PCl:CABG ratio*

\begin{tabular}{|c|c|c|c|c|}
\hline \multirow[b]{2}{*}{ Characteristic } & \multicolumn{4}{|c|}{ Category of hospital PCI:CABG ratio; $\%$ of patients $\dagger$} \\
\hline & $\begin{array}{l}\text { Low } \\
(<2.0)\end{array}$ & $\begin{array}{l}\text { Low-medium } \\
\quad(2.0-2.7)\end{array}$ & $\begin{array}{l}\text { Medium-high } \\
(2.8-3.2)\end{array}$ & $\begin{array}{l}\text { High } \\
(>3.2)\end{array}$ \\
\hline \multicolumn{5}{|l|}{ Patient } \\
\hline No. of patients & 1641 & 2659 & 2833 & 1839 \\
\hline Age, yr, mean (SD) & $65(11)$ & $64(11)$ & $64(12)$ & $64(12)$ \\
\hline Female sex & 34 & 36 & 36 & 34 \\
\hline \multicolumn{5}{|l|}{ Cardiac history and risk factors } \\
\hline Previous $\mathrm{PCl}$ & 12 & 12 & 13 & 18 \\
\hline Diabetes mellitus & 31 & 29 & 26 & 29 \\
\hline Hyperlipidemia & 72 & 69 & 63 & 65 \\
\hline Hypertension & 70 & 69 & 63 & 67 \\
\hline Smoking & 59 & 60 & 48 & 52 \\
\hline \multicolumn{5}{|l|}{ Coronary anatomy, \% } \\
\hline Normal & 19 & 18 & 25 & 20 \\
\hline Mild disease $\neq$ & 9 & 16 & 7 & 7 \\
\hline \multicolumn{5}{|l|}{ Severe stenosis§ } \\
\hline 1 vessel & 26 & 25 & 25 & 29 \\
\hline 2 vessels & 17 & 16 & 18 & 18 \\
\hline 3 vessels & 13 & 11 & 10 & 10 \\
\hline Left main artery & 8 & 5 & 6 & 6 \\
\hline Prior CABG & 8 & 10 & 10 & 9 \\
\hline \multicolumn{5}{|l|}{ Clinical indication for catheterization } \\
\hline $\begin{array}{l}\text { Elective stable coronary artery } \\
\text { disease }\end{array}$ & 40 & 38 & 56 & 43 \\
\hline Unstable angina & 28 & 32 & 18 & 29 \\
\hline Non-ST-segment elevation MI & 20 & 20 & 16 & 15 \\
\hline Non-emergent ST-segment elevation MI & 7 & 5 & 2 & 1 \\
\hline Emergent ST-segment elevation MI & 5 & 6 & 9 & 13 \\
\hline \multicolumn{5}{|l|}{ Physicianๆ } \\
\hline Interventional cardiologist & 39 & 51 & 43 & 44 \\
\hline Non-interventional cardiologist & 60 & 49 & 49 & 56 \\
\hline \multicolumn{5}{|l|}{ Hospital } \\
\hline $\begin{array}{l}\text { Centre performing } \mathrm{PCl} \text { or } \mathrm{CABG} \text {, } \\
\text { or both }\end{array}$ & 66 & 88 & 87 & 93 \\
\hline $\begin{array}{l}\text { Centre performing catheterization } \\
\text { only }\end{array}$ & 34 & 12 & 13 & 7 \\
\hline \multicolumn{5}{|l|}{$\begin{array}{l}\text { Treatment received }<90 \mathrm{~d} \text { after } \\
\text { catheterization }\end{array}$} \\
\hline Medical & 54 & 52 & 52 & 46 \\
\hline $\mathrm{PCl}$ & 28 & 34 & 36 & 44 \\
\hline CABG & 18 & 15 & 12 & 10 \\
\hline Overall PCI:CABG ratio, mean & 1.6 & 2.3 & 3.0 & 4.6 \\
\hline \multicolumn{5}{|c|}{$\begin{array}{l}\text { Note: } \mathrm{CABG}=\text { coronary artery bypass graft, } \mathrm{MI}=\text { myocardial infarction, } \mathrm{PCl}=\text { percutaneous coronary intervention, } \mathrm{SD}=\text { standard } \\
\text { deviation. } \\
\text { *Hospitals were classified into four ratio categories such that there were similar numbers of hospitals (four or five) in each category. } \\
\text { tUnless stated otherwise. The percentages in the physician and hospital sections represent the proportions of patients who } \\
\text { underwent catheterization by the indicated type of cardiologist or hospital. } \\
\text { fStenosis }<70 \% \text { in one or more major epicardial vessels or side branches, or }<50 \% \text { in left main coronary artery. } \\
\text { \$Blockage above thresholds described for mild disease. } \\
\text { IData not available for all patients. }\end{array}$} \\
\hline
\end{tabular}


gent ST-segment elevation MI were greatest in the two highest hospital ratio categories, these patients represented less than $14 \%$ of the patients in any one ratio category. About $45 \%$ of the angiograms in the study were performed by an interventional cardiologist.

Coronary anatomy was the strongest predictor of PCI being chosen over CABG in the multilevel logistic regression model (Table 2). The next two strongest predictors were the clinical indication for the procedure and the hospital where the procedure was performed. Table 3 shows the frequency of PCI among patients who underwent revascularization, by coronary anatomy and category of hospital PCI:CABG ratio. More than $88 \%$ of the patients with single-vessel disease received PCI across the four ratio categories, whereas most patients with left main artery disease underwent CABG surgery regardless of the hospital ratio category. Most of the variation in the PCI:CABG ratio across the four hospital categories occurred among patients with multivessel disease. There was a strong statistical correlation $\left(R^{2}=86 \%\right)$ between a hospital's PCI:CABG ratio among patients with multivessel disease and the centre's overall PCI:CABG ratio.

When we examined the influence of process factors that may have contributed to variations in the PCI:CABG ratio across hospitals, we found a strong association between the treatment recommended by the cardiologist who performed the index catheterization and the type of revascularization therapy the patient ultimately received (Figure 2). Patients whose index catheterization was performed by an interventional cardiologist at a centre in the high PCI:CABG ratio category were more likely to undergo PCI than CABG surgery $(p<0.001$; Figure 3 , top panel). There was also a hospital "cultural" effect, whereby noninterventional cardiologists at centres in the high ratio category were more likely than those at centres in lower ratio groups to select PCI over CABG surgery for their patients $(p<0.001)$. The proportion of PCIs that were performed ad hoc (immediately after the index catheterization) was higher when the index catheterization was done by an interventional cardiologist than when it was done by a noninterventionist $(p<0.001$; Figure 3 , bottom panel); this finding was observed across all hospital ratio categories. Only a small proportion of patients $(<4 \%)$ were discussed at combined (cardiology and surgery) case conferences in any of the hospital ratio categories.

\section{Interpretation}

We observed a threefold variation in the ratio of PCI:CABG procedures across the four hospital groups, from a mean of 1.6 in the group of hospitals with the lowest ratios $(<2.0)$ to a mean of 4.6 in the group with the highest ratios (>3.2), as well as a fivefold variation across the individual hospitals, from 1.3 to 6.1 . The variation was not primarily the result of differences in patient characteristics or the utilization of primary PCI (i.e., for emergent ST-segment elevation myocardial infarction). Rather, it appeared to reflect variations in treatment preferences and practice styles of the cardiologists performing the index catheterizations with regard to the management of patients with non-emergent multivessel disease, patients who could potentially be recommended for either PCI or CABG surgery. ${ }^{17}$ Although coronary anatomy was the most important indi-

Table 2: Significant factors associated with the likelihood of $\mathrm{PCI}$ being performed instead of CABG surgery among patients who underwent revascularizion*

\begin{tabular}{|c|c|c|}
\hline Factor & Odds ratio $(95 \% \mathrm{Cl})$ & $p$ value \\
\hline \multicolumn{3}{|l|}{ Patient } \\
\hline \multicolumn{3}{|l|}{ Age, yr (v. 65-74) } \\
\hline$<50$ & $1.4(0.9-2.0)$ & 0.11 \\
\hline $50-64$ & $1.1 \quad(0.9-1.4)$ & 0.29 \\
\hline$\geq 75$ & $1.6 \quad(1.2-2.1)$ & $<0.001$ \\
\hline Female (v. male) & $1.4(1.1-1.7)$ & 0.003 \\
\hline \multicolumn{3}{|l|}{ Cardiac history and risk factors } \\
\hline Diabetes (v. no diabetes) & $0.8 \quad(0.6-0.9)$ & 0.01 \\
\hline Previous $\mathrm{PCl}$ (v. no previous $\mathrm{PCI}$ ) & $1.6(1.2-2.2)$ & 0.002 \\
\hline \multicolumn{3}{|l|}{ Coronary anatomy (v. 3-vessel disease) } \\
\hline 1 vessel & $37.5(27.9-50.4)$ & $<0.001$ \\
\hline 2 vessels & $5.6(4.5-7.0)$ & $<0.001$ \\
\hline Left main artery & $0.3 \quad(0.2-0.4)$ & $<0.001$ \\
\hline Prior CABG & $30.1(18.7-48.6)$ & $<0.001$ \\
\hline \multicolumn{3}{|l|}{$\begin{array}{l}\text { Clinical indication (v. elective stable } \\
\text { coronary artery disease) }\end{array}$} \\
\hline Unstable angina & $0.9 \quad(0.7-1.2)$ & 0.68 \\
\hline Non-ST-segment elevation MI & $1.3(1.0-1.7)$ & 0.02 \\
\hline Non-emergent ST-segment elevation MI & $1.6(1.0-2.5)$ & 0.04 \\
\hline Emergent ST-segment elevation MI & $7.8 \quad(5.1-11.7)$ & $<0.001$ \\
\hline \multicolumn{3}{|l|}{ Physician } \\
\hline $\begin{array}{l}\text { Interventional (v. non-interventional) } \\
\text { cardiologist }\end{array}$ & $1.4(1.1-1.7)$ & 0.01 \\
\hline \multicolumn{3}{|l|}{ Hospital } \\
\hline \multicolumn{3}{|l|}{ PCI:CABG ratio (v. low ratio $[<2.0]$ ) } \\
\hline Low-medium (2.0-2.7) & $1.4(0.9-2.1)$ & 0.16 \\
\hline Medium-high (2.8-3.2) & $2.1 \quad(1.3-3.1)$ & $<0.001$ \\
\hline High (> 3.2) & $3.1 \quad(2.0-4.9)$ & $<0.001$ \\
\hline \multicolumn{3}{|c|}{$\begin{array}{l}\text { Note: } \mathrm{CABG}=\text { coronary artery bypass graft, } \mathrm{Cl}=\text { confidence interval, } \mathrm{MI}=\text { myocardial } \\
\text { infarction, } \mathrm{PCl}=\text { percutaneous coronary intervention. } \\
\text { *The } \mathrm{C} \text {-statistic for the model was } 0.90 \text {. This analysis excludes patients for whom type of } \\
\text { cardiologist was missing. }\end{array}$} \\
\hline
\end{tabular}




\begin{tabular}{|c|c|c|c|c|c|}
\hline \multirow[b]{2}{*}{$\begin{array}{l}\text { Coronary } \\
\text { anatomy }\end{array}$} & \multicolumn{5}{|c|}{ Hospital PCI:CABG ratio; no. (\%) of patients who underwent $\mathrm{PCl}$ * } \\
\hline & $\begin{array}{l}\text { Low } \\
(<2.0)\end{array}$ & $\begin{array}{l}\text { Low-medium } \\
(2.0-2.7)\end{array}$ & $\begin{array}{l}\text { Medium-high } \\
(2.8-3.2)\end{array}$ & $\begin{array}{l}\text { High } \\
(>3.2)\end{array}$ & Overall \\
\hline 1 vessel & $251(88.4)$ & $422(95.5)$ & $388(92.8)$ & $293(99.0)$ & $1354(94.2)$ \\
\hline 2 vessels & $169(55.0)$ & $303(68.3)$ & $322(76.4)$ & $217(85.7)$ & $1011(72.4)$ \\
\hline 3 vessels & $130(22.3)$ & $204(19.6)$ & $177(35.6)$ & $119(49.6)$ & $630(30.3)$ \\
\hline 2 or 3 vessels & $299(40.8)$ & $507(48.7)$ & $499(61.9)$ & $336(72.9)$ & $1641(56.2)$ \\
\hline Left main artery & $80(11.3)$ & $103(5.8)$ & $104(19.2)$ & $81(14.8)$ & $368(12.8)$ \\
\hline Prior CABG & $43(97.7)$ & 95 (91.6) & $118(89.8)$ & $63(96.8)$ & 319 (92.8) \\
\hline
\end{tabular}

vidual-level predictor of whether a patient received PCI or CABG surgery, there was significant residual variation that could be attributed to hospital and physician factors. These variations in revascularization practice may be clinically significant because the choice of PCI or CABG surgery may result in different long-term outcomes for patients. ${ }^{9-13}$

Our results are consistent with those from previous studies confirming the primary role of the cardiologist who performs the index catheterization in determining the mode of revascularization ultimately provided. Thomp-

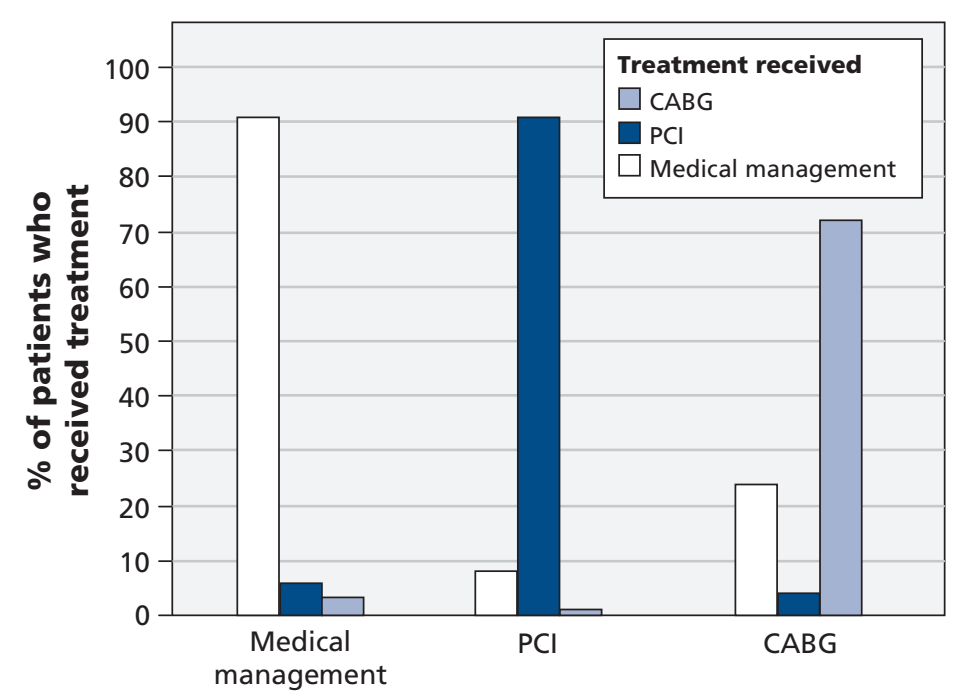

Treatment initially recommended

Figure 2: Treatment initially recommended by the cardiologist performing the index catheterization and the treatment ultimately received within 90 days after the catheterization. The weighted kappa statistic was 0.81 (95\% confidence interval $0.80-0.82$ ) for agreement between the recommended treatment and the treatment received. Excludes 202 patients who died within 90 days after the index catheterization. $\mathrm{CABG}=$ coronary artery bypass graft, $\mathrm{PCl}=$ percutaneous coronary intervention. son and colleagues, using data from coronary angiography registries in the provinces of British Columbia and Alberta, found a similar association between the treatment recommended by the cardiologist immediately after angiography and the treatment received by the patient. ${ }^{18}$ In New York State, Hannan and colleagues observed that many patients who had indications for CABG surgery (according to the American College of Cardiology / American Heart Association guidelines) at the time of angiography received multivessel PCI and never saw a cardiac surgeon. ${ }^{19}$ Although previous studies have shown that interventional cardiologists are more likely to recommend PCI and cardiac surgeons are more likely to recommend $\mathrm{CABG}$ surgery for patients with similar indications, ${ }^{20,21}$ we found substantial variation among cardiology specialists across different hospitals in terms of their revascularization practices.

The trend toward increasing PCI:CABG ratios and increasing use of ad hoc PCI (immediately after cardiac catheterization) have led some observers to raise concerns about potential conflicts of interest, whereby an interventional cardiologist may be in a position both to make a diagnosis and to offer immediate treatment to a patient without involving other members of the health care team such as the referring physician and a cardiac surgeon. ${ }^{22,23}$ Surveys of patients who underwent PCI and CABG surgery showed that more than half of them had not been offered alternative treatment options. ${ }^{23}$ Although emergent treatment with primary PCI is beneficial for patients with ST-segment elevation MI, concerns have been raised about whether the widespread practice of immediate PCI after catheterization in patients with more stable multivessel disease adequately provides for informed con- 
sent. ${ }^{22,23}$ Recent guidelines from the Task Force on Myocardial Revascularization of the European Society of Cardiology and the European Association for Cardio-Thoracic Surgery recommend that ad hoc PCI be avoided in most patients with multivessel disease who are clinically stable. ${ }^{6}$

In our study, patient cases were infrequently discussed at formal, multidisciplinary case conferences. We believe there are opportunities to improve transparency and consistency of decision-making around the mode of revascularization and suggest that an efficient multidisciplinary team approach to decision-making be established for patients who could potentially undergo either PCI or CABG surgery. Such a team could include an interventional cardiologist, the physician who performed the catheterization if not an interventional cardiologist, a cardiac surgeon, the referring physician when feasible, and the patient. Public release of information on the long-term outcomes of PCI and CABG surgery by centre, and increased use of patient decision aids may also help patients make fully informed choices about their treatment options. ${ }^{24}$

\section{Limitations}

Our study has several limitations. First, it was not designed to identify an ideal PCI:CABG ratio. Further study of the outcomes of patients who received treatment at hospitals with different procedure ratios would help identify whether certain practice patterns are associated with better long-term outcomes for patients.

Second, variations in the PCI:CABG ratio could reflect provider knowledge of local outcomes for both procedures. We could not assess whether this was an important consideration from the available data.

Third, we did not have information on the extent to which patients with multivessel disease made a fully informed decision about the choice of PCI versus CABG surgery. However, the high frequency of ad hoc PCI suggests that many of these decisions were likely made without the involvement of a cardiac surgeon.

Fourth, we could not determine from the available data whether there was underuse of PCI among some patients at hospitals with lower PCI:CABG ratios who might have benefitted from PCI.

Finally, variations in the use of medical therapies may have influenced the results of our study. However, a full exploration of this topic was beyond the scope of our study, because we focused only on the utilization of PCI and CABG surgery.

\section{Conclusion}

We found that the recommendation of the physician performing the diagnostic catheterization and the treating hospital were strong independent predictors of the mode of revascularization. Our findings may have important clinical and policy implications and are likely to stimulate debate and discussion about the optimal approach to decisions about the mode of revascularization for patients with advanced coronary artery disease. Although many patients may still prefer PCI because it is less invasive, cardiac surgeons need to be more involved in clinical decision-making when patients are candidates for either PCI or CABG surgery. Patients need to be fully informed about the benefits and risks of all alternative treatment options. Opportunities exist to improve transparency and consistency around the decision-making process for coronary revas-

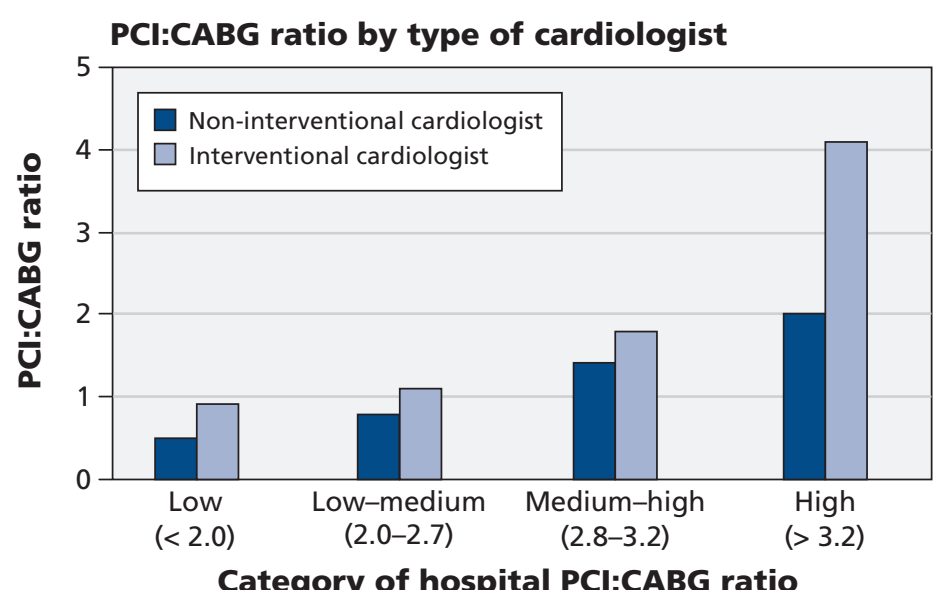

Ad hoc PCls by type of cardiologist

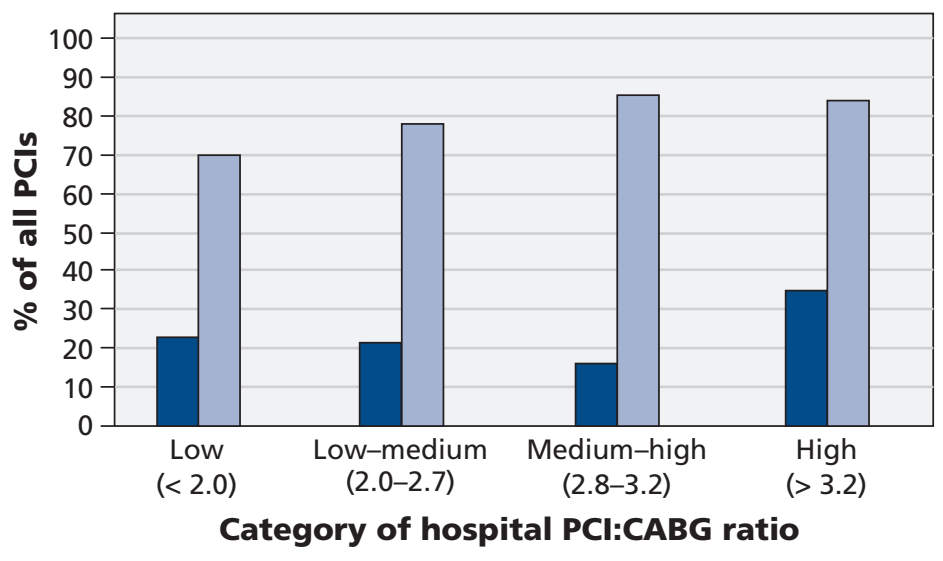

Figure 3: Ratio of percutaneous coronary intervention (PCl) to coronary artery bypass graft (CABG) surgery (top panel) and frequency of ad hoc PCls (performed immediately after the index catheterization) among patients with multivessel disease (bottom panel), by category of hospital PCI:CABG ratio and type of cardiologist who performed the index catheterization. Excludes patients with emergent ST-segment elevation myocardial infarction and patients with cardiogenic shock. 
cularization, most notably among patients with non-emergent multivessel disease.

\section{References}

1. Hassan A, Newman A, Ko DT, et al. Increasing rates of angioplasty versus bypass surgery in Canada, 1994-2005. Am Heart J 2010;160:958-65.

2. Ko DT, Tu JV, Samadashvili Z, et al. Temporal trends in the use of percutaneous coronary intervention and coronary artery bypass surgery in New York State and Ontario. Circulation 2010;121:2635-44.

3. Hannan EL, Wu C, Chassin MR. Differences in per capita rates of revascularization and in choice of revascularization procedure for eleven states. BMC Health Serv Res 2006;6:35.

4. Yusuf S, Zucker D, Peduzzi P, et al. Effect of coronary artery bypass graft surgery on survival: overview of 10-year results from randomised trials by the Coronary Artery Bypass Graft Surgery Trialists Collaboration. Lancet 1994:344:563-70.

5. Patel MR, Dehmer GJ, Hirshfeld JW, et al. ACCF/SCAI/STS/ AATS/AHA/ASNC 2009 Appropriateness Criteria for Coronary Revascularization: A Report of the American College of Cardiology Foundation Appropriateness Criteria Task Force, Society for Cardiovascular Angiography and Interventions, Society of Thoracic Surgeons, American Association for Thoracic Surgery, American Heart Association, and the American Society of Nuclear Cardiology: Endorsed by the American Society of Echocardiography, the Heart Failure Society of America, and the Society of Cardiovascular Computed Tomography. Circulation 2009; 119:1330-52.

6. Wijns W, Kolh P, Danchin N, et al. Guidelines on myocardia revascularization: the Task Force on Myocardial Revascularization of the European Society of Cardiology (ESC) and the European Association for Cardio-Thoracic Surgery (EACTS). Eur Heart J 2010;31:2501-55.

7. Keeley EC, Boura JA, Grines CL. Primary angioplasty versus intravenous thrombolytic therapy for acute myocardial infarction: a quantitative review of 23 randomised trials. Lancet 2003; 361:13-20.

8. Epstein AJ, Polsky D, Yang F, et al. Coronary revascularization trends in the United States, 2001-2008. JAMA 2011;305:1769-76.

9. Serruys PW, Morice MC, Kappetein AP, et al. Percutaneous coronary intervention versus coronary-artery bypass grafting for severe coronary artery disease. N Engl J Med 2009;360:961-72.

10. Bravata DM, Gienger AL, McDonald KM, et al. Systematic review: the comparative effectiveness of percutaneous coronary interventions and coronary artery bypass graft surgery. Ann Intern Med 2007;147:703-16.

11. Hlatky MA, Boothroyd DB, Bravata DM, et al. Coronary artery bypass surgery compared with percutaneous coronary interventions for multivessel disease: a collaborative analysis of individual patient data from ten randomised trials. Lancet 2009;373:1190-7.

12. Hannan EL, Racz MJ, Walford G, et al. Long-term outcomes of coronary-artery bypass grafting versus stent implantation. N Engl J Med 2005;352:2174-83.

13. Hannan EL, Wu C, Walford G, et al. Drug-eluting stents vs. coronary-artery bypass grafting in multivessel coronary disease. N Engl J Med 2008;358:331-41.

14. Gurevich Y, McFarlane A, Morris K, et al. Estimating the number of coronary artery bypass graft and percutaneous coronary intervention procedures in Canada: a comparison of cardiac registry and Canadian Institute for Health Information data sources. Can J Cardiol 2010;26:e249-53.

15. Austin PC, Goel V, van Walraven C. An introduction to multilevel regression models. Can J Public Health 2001;92:150-4.

16. Tu JV, Willison DJ, Silver FL, et al. Impracticability of informed consent in the Registry of the Canadian Stroke Network. $N$ Engl $J$ Med 2004;350:1414-21.

17. Naylor CD. Grey zones of clinical practice: some limits to evidence-based medicine. Lancet 1995;345:840-2.

18. Thompson CR, Humphries KH, Gao M, et al. Revascularization use and survival outcomes after cardiac catheterization in British Columbia and Alberta. Can J Cardiol 2004;20:1417-23.

19. Hannan EL, Racz MJ, Gold J et al. Adherence of catheterization laboratory cardiologists to American College of Cardiology / American Heart Association guidelines for percutaneous coronary interventions and coronary artery bypass graft surgery: What happens in actual practice? Circulation 2010;121:267-75.

20. Bernstein SJ, Lazaro P, Fitch K, et al. Effect of specialty and nationality on panel judgments of the appropriateness of coro- nary revascularization: a pilot study. Med Care 2001;39:513-20.

21. Denvir MA, Pell JP, Lee AJ et al. Variations in clinical decisionmaking between cardiologists and cardiac surgeons: A case for management by multidisciplinary teams? J Cardiothorac Surg 2006; $1: 2$.

22. Nallamothu BK, Krumholz HM. Putting ad hoc PCI on pause. JAMA 2010;304:2059-60.

23. Chandrasekharan DP, Taggart DP. Informed consent for interventions in stable coronary artery disease: problems, etiologies, and solutions. Eur J Cardiothorac Surg 2011;39:912-7.

24. Morgan MW, Deber RB, Llewellyn-Thomas HA, et al. Randomized, controlled trial of an interactive videodisc decision aid for patients with ischemic heart disease. J Gen Intern Med 2000;15: 685-93.

Affiliations: From the Institute for Clinical Evaluative Sciences (Tu, Ko, Guo, Richards), Toronto, Ont.; the Sunnybrook Schulich Heart Centre (Tu, Ko, Wijeysundera, Cohen), Toronto, Ont.; the University of Toronto ( $\mathrm{Tu}, \mathrm{Ko}, \mathrm{Guo}$, Wijeysundera, Latter, Feindel, Cohen), Toronto, Ont.; Ryerson University (Walton), Toronto, Ont.; Hamilton Health Sciences Centre, McMaster University (Natarajan), Hamilton, Ont.; the University of Ottawa Heart Institute (So), Ottawa, Ont.; St. Michael's Hospital (Latter), Toronto, Ont.; the University Health Network (Feindel), Toronto, Ont.; and the Cardiac Care Network of Ontario (Kingsbury), Toronto, Ont.

Contributors: All of the authors made substantial contributions to the conception and design of this project and participated in the acquisition of data, and in the analysis and interpretation of the data. Jack Tu drafted the article and all of the other authors participated in revising it for important intellectual content. All of the authors have approved the final version of the article to be published. Jack Tu had full access to all of the data in the study and takes responsibility for the integrity of the data and the accuracy of the data analysis.

Funding: This project was supported by an operating grant from the Ontario Ministry of Health and Long-Term Care and a Canadian Institutes of Health Research (CIHR) Team Grant in Cardiovascular Outcomes Research. The Cardiac Care Network of Ontario and the Institute for Clinical Evaluative Sciences are funded by the Ontario Ministry of Health and LongTerm Care. Jack Tu is supported by a Canada Research Chair in Health Services Research and a Career Investigator Award from the Heart and Stroke Foundation of Ontario. Dennis Ko is supported by a CIHR New Investigator Award.

Acknowledgements: The authors thank all of the administrators and clinicians at the member hospitals of the Cardiac Care Network of Ontario who helped facilitate completion of this study at their respective institutions.

The results and conclusions are those of the authors and should not be attributed to any of the funding or participating organizations.

Members of the Variations in Revascularization Practice in Ontario Working Group: Drs. Jack V. Tu and Eric A. Cohen (co-chairs), Sunnybrook Schulich Heart Centre, Toronto, Ont.; Dr. Christopher M. Feindel, University Health Network, Toronto, Ont.; Helen Guo, Institute for Clinical Evaluative Sciences, Toronto, Ont.; Dr. Ansar Hassan, Saint John Regional Hospital, Saint John, NB; Kori Kingsbury, Cardiac Care Network of Ontario, Toronto, Ont.; Dr. Dennis T. Ko, Sunnybrook Schulich Heart Centre, Toronto, Ont.; Dr. David A. Latter, St. Michael's Hospital, Toronto, Ont.; Dr. A. Dave Nagpal, London Health Sciences Centre, London, Ont.; Dr. Madhu K. Natarajan, Hamilton Health Sciences Centre, McMaster University, Hamilton, Ont.; Dr. Joseph Noora, Trillium Health Centre, Mississauga, Ont.; Janice A. Richards, Institute for Clinical Evaluative Sciences, Toronto, Ont.; Heather Sherrard and Dr. Derek So, University of Ottawa Heart Institute, Ottawa, Ont.; Nancy Walton, Ryerson University, Toronto, Ont.; and Dr. Harindra C. Wijeysundera, Sunnybrook Schulich Heart Centre, Toronto, Ont. 